\title{
КОМПЛЕКСНОЕ ЛЕЧЕНИЕ ВНУТРЕННИХ НАРУШЕНИЙ ВИСОЧНО-НИЖНЕЧЕЛЮСТНОГО СУСТАВА С ПРИМЕНЕНИЕМ ОККЛЮЗИОННОЙ ШИНОТЕРАПИИ, АРТРОЦЕНТЕЗА И АРТРОСКОПИИ
}

\begin{abstract}
COMPLEX TREATMENT
OF INTERNAL DISORDERS

OF THE TEMPOROMANDIBULAR JOINT

USING OCCLUSIVE SPLINT THERAPY, ARTHROCENTESIS AND ARTHROSCOPY

\section{B. Gharamyan \\ T. Chkhikvadze \\ V. Bekreev}

Summary. Diseases of the temporomandibular joint are complex and polyetiologic disorders. Among these diseases, the first place in terms of distribution is occupied by internal disorders of the temporomandibular joint (ID TMJ), which mean intra-articular changes, both anatomical and functional. Treatment of ID TMJ requires the use of a whole arsenal of methods. A staged treatment was carried out, including occlusive splint therapy (stage I), with insufficient effectiveness of occlusive splint therapy, additional arthrocentesis (stage II), occlusive splint therapy and arthrocentesis in combination with arthroscopy (stage III of treatment) were included. As a result of the use of complex treatment, the normalization of TMJ function was achieved in $98,44 \%$ of patients, the rest showed a significant improvement.
\end{abstract}

CO
OF
OF
US
AR
sum
poly
distr
joint
and
of
splin
splint
thera
treat
norm
rest
Key
arth

Keywords: temporomandibular joint, TMJ, occlusive splint therapy, arthrocentesis, arthropuncture, arthroscopy.

\section{Ввеление}

исочно-нижнечелюстной сустав (ВНЧС) парный, синовиальный, обеспечивающий подвижность нижней челюсти, представляет собой сложную биомеханическую систему. В ВНЧС различают два суставных пространства, верхнее и нижнее, разделенных суставным диском [14].

ВН ВНЧС - группа темпоромандибулярных расстройств ВНЧС, возникающих при нарушении анатомических и функциональных взаимоотношений внутрисуставных элементов. Чаще всего они встречаются при
Гарамян Борис Георгиевич

Аспирант, ФГАОУ ВО "Российский университет дружбы народов" garamjan13@gmail.com

чхиквадзе Тина Владимировна Аспирант, ФГАОУ ВО "Российский университет дружбы народов" tchkhik@hotmail.com

Бекреев Валерий Валентинович Д.м.н., профессор, ФГАОУ ВО "Российский университет дружбы народов" bekreev@mail.ru

Аннотация. Заболевания височно-нижнечелюстного сустава — сложные и полиэтиологичные расстройства. Среди этих заболеваний первое место по распространению занимают внутренние нарушения височно-нижнечелюстного сустава (ВН ВНЧС), под которыми подразумеваются внутрисуставные изменения, как анатомические, так и функциональные. Лечение ВН ВНЧС требует применения целого арсенала методов. Проводилось этапное лечение, включающее в себя окклюзионную шинотерапию (I этап), при недостаточной эффективности окклюзионной шинотерапии включили дополнительно артроцентез (ІІ этап) и окклюзионную шинотерапию и артроцентез В сочетании с артроскопией (III этап лечения). В результате применения комплексного лечения нормализация функции ВНЧС была достигнута у 98,44\% пациентов, у остальных наблюдалось значительное ее улучшение.

Ключевые слова: височно-нижнечелюстной сустав, ВНЧС, окклюзионная шинотерапия, артроцентез, артропункция, артроскопия.

смещении суставного диска, его деформации, растяжении или разрыве внутрисуставных связок $[1 ; 8]$.

В разных странах стоматологи отмечают рост обращений пациентов за лечебной помощью с ВН ВНЧС. Внутренние нарушения чаще встречаются у женщин, чем у мужчин. Это объясняется повышенным уровнем гормона эстрогена и, кроме того, большим влиянием эмоциональных факторов у женщин по сравнению с мужчинами $[4 ; 12 ; 15]$.

Этиология ВН ВНЧС сложна и многофакторна, разные авторы предлагают разные теории, основные - это ок- 
клюзионно-артикуляционная, миогенная, психоэмоциональная, суставная теории [4; 12].

Диагностика ВН ВНЧС требует тщательного сбора анамнеза и применения современных средств визуализации мягкотканых элементов. Считается, что «золотым стандартом» визуализации мягких тканей при ВН ВНЧС является магнито-резонансная томография (МРТ) ВН ВНЧС. По данным В.В. Бекреева с соавт. (2016), доступный и безопасный метод ультрозвуковая диагностика дает возможность визуализации головки нижней челюсти, суставного диска, капсулярно-связочного и мышечного аппарата ВНЧС и использовать его для контроля эффективности лечения. Компьютерная томография применяется для диагностики реже, поскольку при ней не визуализирует суставный диск, а костные изменения на ранних стадиях ВН ВНЧС, как правило, отсутствуют. Рентгенологическое исследование эффективно при первичных и вторичных остеоартрозах $[1 ; 7 ; 9]$.

Использование окклюзионных шин в лечении ВН ВНЧС позволяет во многих случаях достичь нормализации анатомических и функциональных взаимоотношений внутрисуставных структур, а также предупредить развитие вторичного остеоартроза и рецидива смещения суставного диска. Другие пациенты, у которых окклюзионная шинотерапия была недостаточно эффективной, нуждаются в хирургическом вмешательстве [6; 16].

Существует широкий спектр хирургических процедур при ВН ВНчС, начиная от артроцентеза височно-нижнечелюстного сустава и артроскопии и заканчивая открытыми хирургическими операциями - артротомиями $[10 ; 11]$.

Артроцентез - несложный и эффективный метод лечения, который показан в случаях, когда окклюзионная шинотерапия неэффективна [2; 11$]$.

При неэффективности окклюзионной шинотерапии и артроцентеза применяют артроскопию. Артроскопия позволяет провести прямой осмотр внутренних структур ВНЧС с помощью эндоскопа. Назначаемая по показаниям пациентам артроскопия как диагностическая процедура при выявлении патологии в процессе проведения может приобрести лечебный характер. Авторы также указывают на успешное применение в последние годы комбинированного лечения ВН ВНЧС, эффективность которого составляет по разным оценкам 90-97\% $[2 ; 12 ; 13]$.

\section{Цель исслеАования}

Целью настоящего исследования являлось определение эффективности этапного применения шинотера- пии, артроцентеза и артроскопии при внутренних нарушениях височно-нижнечелюстного сустава.

\section{Материалы и метолы исслеАования}

Клиническое обследование пациентов проводилось на базе МЦ РУДН.

Было обследовано 64 пациента с двусторонним Внутренним нарушением ВНЧС. В зависимости от клинической формы ВН ВНЧС (согласно классификации П.Г. Сысолятина и др. [3]) пациенты были разделены на 3 группы: 1) с подвывихом суставного диска; 2) с хроническим вывихом суставного диска с частичной репозицией и 3) с хроническим вывихом суставного диска без репозиции.

Всем пациентам было проведено клиническое и дополнительное обследование (МРТ ВНЧС, УЗИ ВНЧС, КЛКТ головы, ОПТГ). Для оценки интенсивности боли использовали визуально-аналоговую шкалу. Оценивалась степень открывания рта. Динамика устранения деформации суставного диска оценивалась измерением толщины различных частей суставного диска и подвижности суставного диска с помощью УЗИ ежемесячно, а также проводилось МРТ ВНЧС до и после лечения.

Лечение пациентов с ВН ВНЧС проводилось в три этапа. Первый этап заключался в проведении окклюзионной шинной терапии, которая применялась в течение 3 месяцев. Если это было неэффективно или малоэффективно, проводился второй этап лечения: окклюзионную шинотерапию применяли совместно с артроцентезом. В тех случаях, когда второй этап не приносил не приносил желаемых результатов, на третьем этапе наряду с вышеуказанными методами применялась артроскопия.

Результаты исследования (цифровые значения, отражающие положение, подвижность и степень деформации диска, полученным при УзИ и МРТ ВНЧС) были статистически обработаны с помощью однорангового критерия Вилкоксона $(p<0,01)$. Различия между группами по среднему значению максимального открывания рта оценивались с помощью критерия хи-квадрат $(p<0,05)$.

\section{Результаты и обсужление}

Одним из критериев эффективности лечения пациентов с ВН ВНЧС является степень открывания рта.

На рисунке 1 отражены средние показатели величины максимального открывания рта на трех этапах до и после проведенного лечения. 


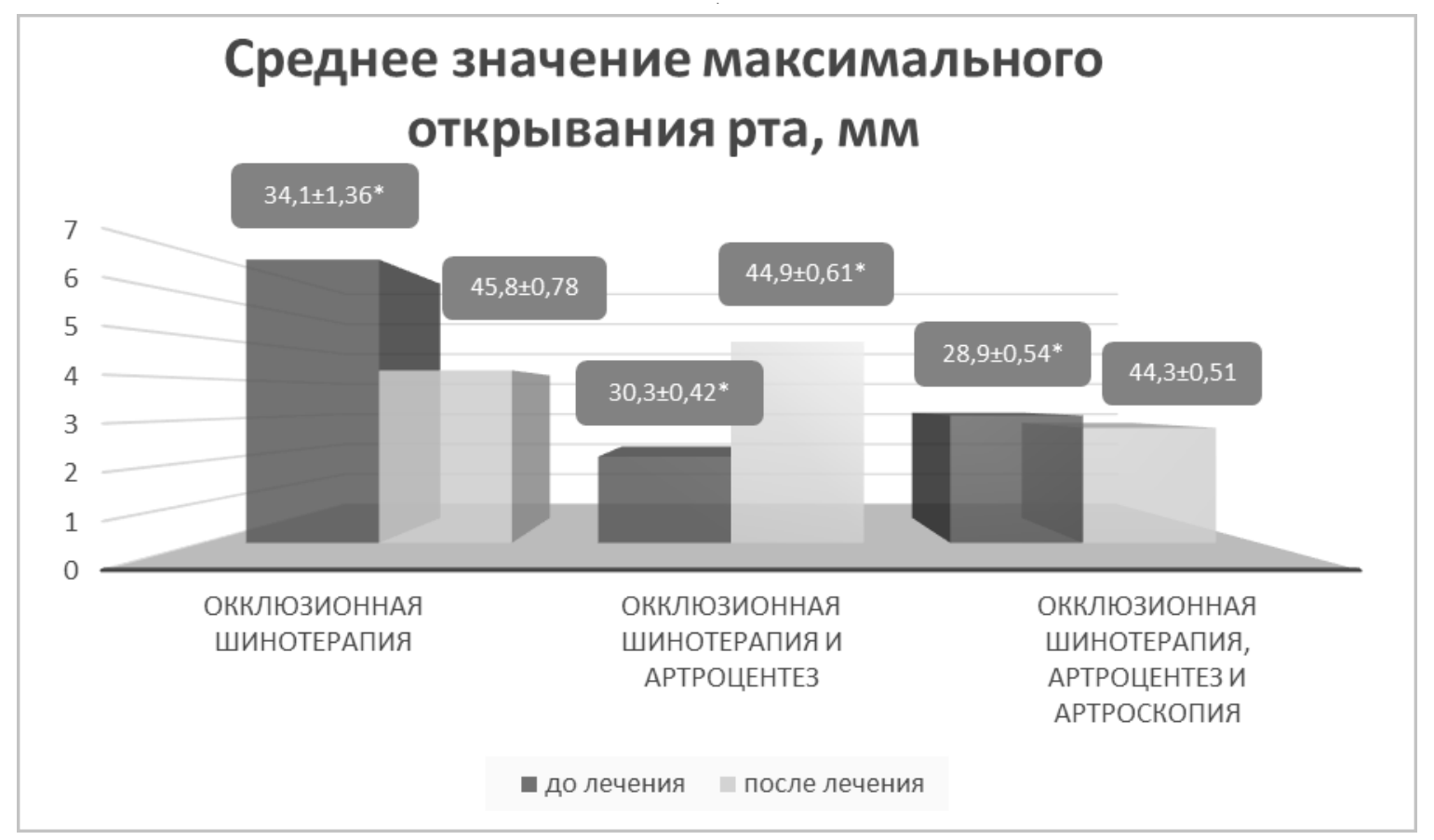

Рис. 1. Среднее значение максимального открывания рта ( $\left.{ }^{*} p<0,05\right)$

На первом этапе (окклюзионная шинотерапия) средняя величина максимального открывания рта перед лечением составила $34,1 \pm 1,36^{*}$ мм, после лечения $45,8 \pm 0,78$ мм, перед лечением на втором этапе (окклюзионная шинотерапия и артроцентез) - 30,3 $30,42 *$

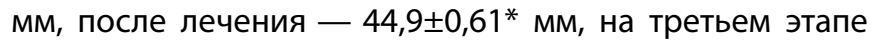
(окклюзионная шинотерапия, артроцентез и артроскопия) перед лечением показатель находился у отметки

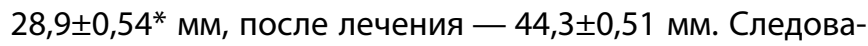
тельно, у пациентов на всех этапах лечения при применении ОШ, а также в комбинации ее с артроцентезом и артроскопией получена нормализация степени открывания рта.

Средняя продолжительность лечения составила на 1 этапе, где применялась только окклюзионная шинотерапия 6,8 месяцев, на втором этапе (окклюзионная шинотерапия и артроцентез) - 8,5 месяцев и на 3 этапе (окклюзионная шинотерапия, артроцентез и артроскопия) $-12,7$ месяцев.

Объективным показателем нормализации функции ВНЧС является устранение деформации суставного диска, подтвержденное измерением толщины разных его частей при УЗИ.

На рисунке 2 показано, сколько пациентов поступило на каждый этап лечения и сколько достигло нормализации положения и функции суставного диска по результатам УЗИ.
Так, на первом этапе из 64 (100\%) пациентов нормализации положения и функции суставного диска достигнуто у $17(27,87 \%)^{* *}$; на втором этапе из $47(100 \%)^{* *}$ пациентов нормализация наступила у 41 (87,23\%), а на третьем этапе вылечились 5 (83,33\%) человек, у 1 пациента полной нормализации функции ВНЧС не наступило, ему была рекомендована повторная артроскопия. Результаты исследования показывают, что для всех пациентов лечение нужно начинать с окклюзионной шинотерапии как базового метода лечения. Если эффективность не достигается на этом этапе лечения, то необходимо прибегнуть к применению артроцентеза (второй этап лечения) в сочетании с окклюзионной шинотерапией. В случае неэффективности второго этапа лечения, применяется артроскопия. На каждом этапе лечения ежемесячно проводятся ультразвуковые исследования и МРТ в начале и после лечения.

В настоящем исследовании нормализация положения и функции суставного диска достигнута у 98,44\% (63 пациента), у остальных 1,56\% (1 пациент) наблюдалось значительное улучшение функции ВНЧС. Данные результаты свидетельствуют о целесообразности применения поэтапного лечения ВН ВНЧС.

\section{Выво $\triangle$}

Проведение окклюзионной шинной терапии при лечении пациентов с ВН ВНЧС приводит к нормализации положения и функции суставного диска в 27,87\% случаев. В случаях малоэффективности и неэффективности 


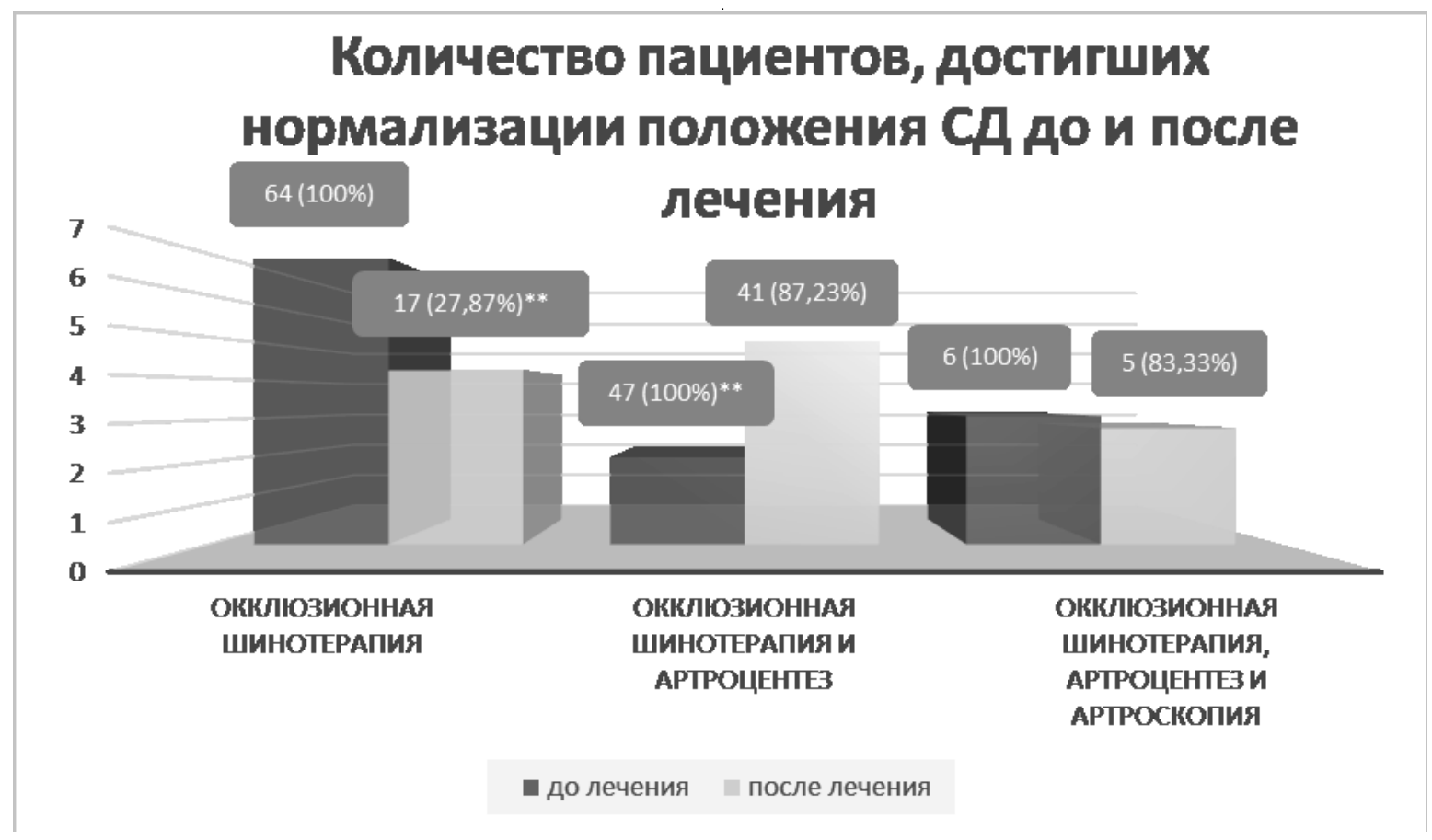

Рис. 2. Количество пациентов, достигших нормализации положения СД до и после лечения на трех этапах лечения (** $\mathrm{p}<0,01)$

пациентам показаны хирургические малоинвазивные методы лечения: артроцентез в сочетании с шинотерапией (эффективность лечения в данном исследовании составила 87,23\%) и артроскопия в сочетании с артро- центезом и шинотерапией (эффективность лечения 83,33\%). Результаты исследования свидетельствуют о целесообразности поэтапного применения указанных методов лечения.

\section{ЛИТЕРАТУРА}

1. Бекреев В.В., Иванов С.Ю., Буренчев Д.В., Груздева Т.А., Юркевич Р.И., Гарамян Б.Г. Применение ультразвукового метода исследования в диагностике внутренних нарушений ВНЧС. Медицинский алфавит. 2016; 29 (4): 37-41.

2. Ильин, А.А. Артроцентез височно-нижнечелюстного сустава. Обзор литературы /А.А. Ильин, А.В. Адоньева // Journal of Siberian Medical Sciences. 2014. 一 № 6. - С. 36.

3. Сысолятин, П.Г. Классификация заболеваний и повреждений височнонижнечелюстного сустава / П.Г. Сысолятин, А.А. Ильин, П. Дергилев.- М.: Медицинская книга.—Н. Новгород: Издательство НГМА, 2000.— 79 с.

4. Фадеев Р.А., Кудрявцева 0.А., Польщикова И.В. Выявление и подготовка к устранению окклюзионных нарушений у пациентов с дисфункциями височно-нижнечелюстных суставов (Ч. 2) // Институт стоматологии. 2016. № 4(33). С.38-39.

5. Ян, Ч. Оценка окклюзионных шин при репозиции переднего вывиха диска височно-нижнечелюстного сустава с репозицией: наблюдение от 3 до 36 месяцев /Ч. Ян, П. Шэнь // Альманах клинической медицины. — 2017.— № 6.— C. 478-485.

6. Ян, Ч. Комбинированное лечение «Сустав — нижняя челюсть — окклюзия»: новая теория и наш протокол/Ч. Ян, Д. Хэ, М. Чэнь [и др.] // Альманах клинической медицины.— 2017.— № 6.- С. 440-459.

7. Al-Saleh, M.A. MRI and CBCT image registration of temporomandibular joint: A systematic review / M.A. Al-Saleh, N.A. Alsufyani, H. Saltaji [et al] // J. Otolaryngol. Head Neck Surg. — 2016. - 45. - P. 30; doi:10.1186/s40463-016-0144-4.

8. Azhar, F. Multiple etiologies of temporomandibular joint disorders -a review / F. Azhar, A. Sani, S. Baig // Pakistan journal of medicine and dentistry. — 2019. — Vol. $8(03)$ - P.53-57.

9. Berg, T. Correlation between objective clinical symptoms and different MRI sequences in diseases of the temporomandibular joint/T. Berg, A. Rahmiel // Conference: 15th Biennial Congress of the European Dental Association. - 2021.

10. Forssell, H. Explanatory Models of Illness and Treatment Goals in Temporomandibular Disorder Pain Patients Report-ing Different Levels of Pain-Related Disability / H. Forssell, U. Kotiranta, T. Kauko [et al] // J Oral Facial Pain Headache. — 2016. — 30(1). — P.14-20. 
11. Guarda-Nardini, L. Arthrocentesis of the Temporomandibular Joint: Systematic Review and Clinical Implications of Research Findings / L. Guarda-Nardini, A.M. De Almeida, D. Manfredini. //J Oral Facial Pain Headache. - 2021. - 35(1). - P.17-29.

12. Li, D.T.S. Temporomandibular Disorders: Current Concepts and Controversies in Diagnosis and Management/ D.T.S. Li, Y.Y. Leung // Diagnostics — 2021. — 11. — P. 459; https://doi.org/10.3390/ diagnostics11030459.

13. List, T. Temporomandibular disorders: old ideas and new concepts/T. List, R.H. Jensen//Cephalalgia. — 2017. — 37.—P.692-704, doi:10.1177/0333102416686302.

14. Okeson, J.P. Orthodontic therapy and the patient with temporomandibular disorder. / J.P. Okeson //Orthodon-tics-E-Book: Current Principles and Techniques. 2016. - P. 353-366.

15. Osiewicz, M.A. Frequency of temporomandibular disorders diagnosesbased on RDC/TMD in a Polish patient population / M.A. Osiewicz, F. Lobbezoo, B.W. Loster // CRANI0 ${ }^{\circledR}$. 2017.-36.- P.1-7.

16. Santhosh Kumar, M.P. Surgical Treatment Modalities in the Management of Temporomandibular Joint Disorders/ M.P. Santhosh Kumar, K. Murugesan, N. Hinaz // Int J Dentistry Oral Sci.— 2021.— 8(8). - P.4168-4179.

( Гарамян Борис Георгиевич ( garamjan13@gmail.com ),

Чхиквадзе Тина Владимировна ( tchkhik@hotmail.com ), Бекреев Валерий Валентинович ( bekreev@mail.ru ).

Журнал «Современная наука: актуальные проблемы теории и практики»

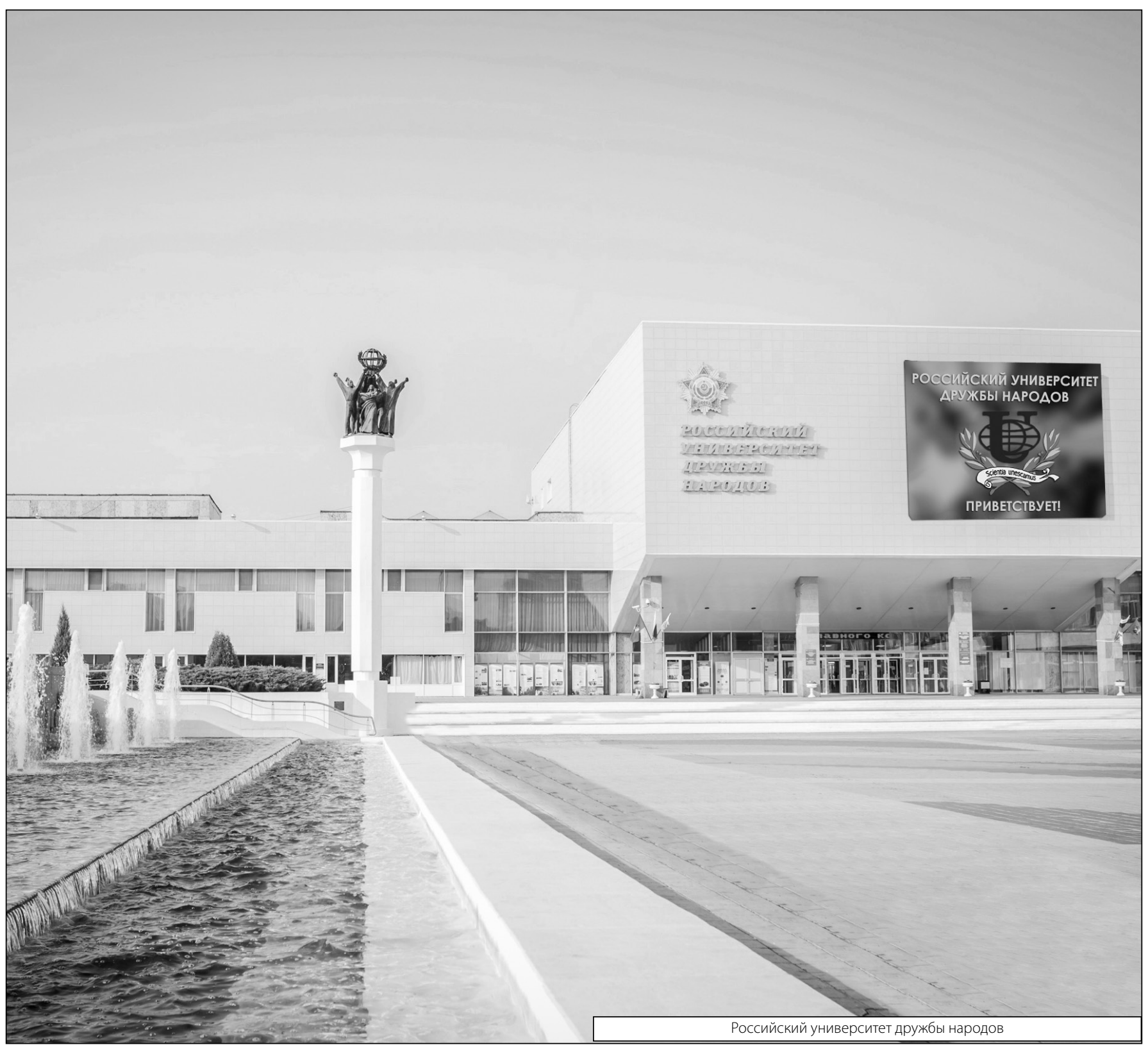

